University of Wollongong

Research Online

Faculty of Informatics - Papers (Archive)

Faculty of Engineering and Information

Sciences

May 2000

\title{
Computing running DCTs and DSTs based on their second-order shift properties
}

Jiangtao Xi

University of Wollongong, jiangtao@uow.edu.au

Joe F. Chicharo

University of Wollongong, chicharo@uow.edu.au

Follow this and additional works at: https://ro.uow.edu.au/infopapers

Part of the Physical Sciences and Mathematics Commons

\section{Recommended Citation}

$\mathrm{Xi}$, Jiangtao and Chicharo, Joe F.: Computing running DCTs and DSTs based on their second-order shift properties 2000.

https://ro.uow.edu.au/infopapers/13

Research Online is the open access institutional repository for the University of Wollongong. For further information contact the UOW Library: research-pubs@uow.edu.au 


\title{
Computing running DCTs and DSTs based on their second-order shift properties
}

\author{
Abstract \\ This paper presents a set of second-order recursive equations which are referred to as the second-order \\ shift (SOS) properties of the discrete cosine transform (DCT) and the discrete sine transform (DST). The \\ proposed SOS properties enable independent updating of the respective DCT and DST coefficients. This \\ is in direct contrast with existing methodology for computing the running DCT and DST where there is an \\ inherent interdependency between the DCT and DST coefficients. The SOS properties provide more \\ efficient algorithms in terms of computational burden and memory requirements when implementing \\ running DCTs and DSTs. \\ Keywords \\ DCT, discrete cosine transform, discrete sine transform \\ Disciplines \\ Physical Sciences and Mathematics

\section{Publication Details} \\ This article was originally published as: Xi, J \& Chicharo, JF, Computing running DCTs and DSTs based on \\ their second-order shift properties, IEEE Transactions on Circuits and Systems-I: Fundamental Theory and \\ Applications, May 2000, 47(5), 779-783. Copyright IEEE 2000.
}




\section{REFERENCES}

[1] B. Razavi, "Challenges in portable RF transceiver design," IEEE Circuits Devices, pp. 12-26, Sept. 1996.

[2] F. H. Raab, "Idealized operation of the class E tuned power amplifier," IEEE Trans. Circuits Syst., vol. CAS-24, pp. 725-735, Dec. 1977.

[3] - "Effects of variations on the class E tuned power amplifier," IEEE J. Solid-State Circuits, vol. SC-13, pp. 239-247, Apr. 1978.

[4] J. A. Blanchard and J. S. Yuan, "Effects of collector current exponential decay on power efficiency for class E tuned power amplifier," IEEE Trans. Circuits Syst.I, vol. 41, pp. 69-72, Jan. 1994.

[5] M. J. Chudobiak, "The use of parasitic nonlinear capacitors in class E amplifiers," IEEE Trans. Circuits Syst. I, vol. 41, pp. 941-944, Dec. 1994.

[6] T. Sowlati et al., "Low voltage, high efficiency GaAs class E power amplifier for wireless transmitters," IEEE J. Solid-State Circuits, vol. 30, pp. 1074-1080, Oct. 1995

[7] N. O. Sokal and F. H. Raab, "Harmonic output of class E RF power amplifier and load coupling network design," IEEE J. Solid-State Circuits, vol. SC-12, pp. 86-88, Feb. 1977.

[8] N. M. Nguyen and R. G. Meyer, "Si IC-compatible inductors and LC passive filters," IEEE J. Solid-State Circuits, vol. 25, pp. 1028-1031, Aug. 1990.

[9] C. Li and Y. Yam, "Maximum frequency and optimum performance of class E power amplifiers," Proc. Inst. Elect. Eng., vol. 141, no. 3, June 1994.

[10] J. Chang, A. A. Abidi, and M. Gaitan, "Large suspended inductors on silicon and their use in a $2-\mu \mathrm{m}$ CMOS RF amplifier," IEEE Electron. Device Lett., vol. 14, May 1993.

[11] N. O. Sokal and A. D. Sokal, "Class E, a new class of high efficiency tuned single-ended switching power amplifiers," IEEE J. Solid-State Circuits, vol. SC-10, pp. 168-176, June 1975.

\section{Computing Running DCT's and DST's Based on Their Second-Order Shift Properties}

Jiangtao Xi and Joe F. Chicharo

\begin{abstract}
This paper presents a set of second-order recursive equations which are referred to as the second-order Shift (SOS) properties of the discrete cosine transform (DCT) and the discrete sine transform (DST). The proposed SOS properties enable independent updating of the respective DCT and DST coefficients. This is in direct contrast with existing methodology for computing the running DCT and DST where there is an inherent interdependency between the DCT and DST coefficients. The SOS properties provide more efficient algorithms in terms of computational burden and memory requirements when implementing running DCT's and DST's.
\end{abstract}

Index Terms-DCT, discrete cosine transform, DST.

\section{INTRODUCTION}

The discrete cosine transform (DCT) [1] has been successfully applied to the fields of speech and image processing. In order to compute the DCT efficiently, various fast and efficient block- based algorithms have been proposed (for example, see [2]-[5] and their references). However, in the case where the DCT parameters need to be updated for every new signal sample, the running DCT implementation

Manuscript received September 16, 1997; revised March 6, 1999. This paper was recommended by Associate Editor J. Götze.

The authors are with the School of Electrical, Computer, and Telecommunications Engineering, The University of Wollongong, Wollongong, NSW 2522, Australia

Publisher Item Identifier S 1057-7122(00)03966-0. is required. As indicated by [7], the computational burden for the running DCT is rather intensive. One approach for computing the running DCT's and discrete sine transforms (DST's) is to use their shift properties, as derived by Yip and Rao [6]. The shift properties are a set of recursive equations that can be used for updating the DCT's and DST's coefficients. However, this approach is not very efficient in terms of computation. A source of the excessive computational burden is the dependency between a DCT coefficient and its corresponding DST coefficient. The process of updating a DCT (or DST) requires updating the corresponding DST (or DCT). To alleviate this problem, Murthy and Swamy [7] proposed an approach for DCT-II, DST-II, DCT-IV, and DST-IV. In [7] each transform member was represented as the real part of a complex function and recursive equations were derived for updating these complex functions. In other words, the approach updates complex functions rather than transform coefficients. It is obvious that there is still some excessive and unnecessary computation.

This paper proposes a more efficient class of running algorithms for DCT's and DST's by deriving a set of recursive equations which enable the independent updating of each DCT and DST member respectively. The proposed recursive equations are called second-order shift (SOS) properties due to their second-order nature. By contrast, the shift properties derived by Yip and Rao [6] are referred to as the first-order shift (FOS) properties, as they are in effect first-order recursive equations.

This paper is organized as follows.: Section II reviews the FOS properties of DCT's and DST's. The SOS properties for the DCT's and DST's are derived and presented in Section III. A performance analysis of running DCT's and DST's based on SOS properties is undertaken in Section IV and the results are compared with the most recent approaches reported in literature thus far. Finally, Section V concludes the paper.

\section{FIRST-ORDER ShIFT PROPERTIES OF DCT's AND DST's}

As defined in [8], the family of DCT's and DST's for the signal block $x(n-N), x(n-N+1), \cdots, x(n)$ is given as follows [8]

DCT-I:

$\begin{aligned} c(n, k)=\sqrt{\frac{2}{N}} P_{k} \sum_{m=0}^{N} P_{m} x(n-N+m) \cos \left(\frac{m \pi}{N} k\right), & \\ & \text { for } k=0,1, \cdots, N\end{aligned}$

\section{DCT-II}

$c(n, k)=\sqrt{\frac{2}{N}} P_{k} \sum_{m=0}^{N-1} x(n-N+m) \cos \left\{\left(m+\frac{1}{2}\right) k \frac{\pi}{N}\right\}$,

for $k=0,1, \cdots, N-1$
DCT-III: $c(n, k)=\sqrt{\frac{2}{N}} \sum_{m=0}^{N-1} P_{m} x(n-N+m) \cos \left\{m\left(k+\frac{1}{2}\right) \frac{\pi}{N}\right\}$, for $k=0,1, \cdots, N-1$

\section{DCT-IV:}

$c(n, k)=\sqrt{\frac{2}{N}} P_{k} \sum_{m=0}^{N-1} x(n-N+m) \cos \left\{\left(m+\frac{1}{2}\right)\left(k+\frac{1}{2}\right) \frac{\pi}{N}\right\}$, for $k=0,1, \cdots, N-1$ 
and

DST-I:

$s(n, k)=\sqrt{\frac{2}{N}} \sum_{m=0}^{N} x(n-N+m) \sin \left(\frac{m \pi}{N} k\right)$,

$$
\text { for } k=1,2, \cdots, N-1
$$

DST-II:

$s(n, k)=\sqrt{\frac{2}{N}} P_{k} \sum_{m=0}^{N} x(n-N+m) \sin \left\{\left(m-\frac{1}{2}\right) k \frac{\pi}{N}\right\}$,

for $k=1,2, \cdots, N$

DST-III:

$s(n, k)=\sqrt{\frac{2}{N}} \sum_{m=0}^{N} P_{m} x(n-N+m) \sin \left\{m\left(k+\frac{1}{2}\right) \frac{\pi}{N}\right\}$,

$$
\text { for } k=1,2, \cdots, N
$$

DST-IV:

$s(n, k)=\sqrt{\frac{2}{N}} \sum_{m=0}^{N-1} x(n-N+m) \sin \left\{\left(m+\frac{1}{2}\right)\left(k+\frac{1}{2}\right) \frac{\pi}{N}\right\}$, for $k=0,1, \cdots N-1$

where

$$
P_{j}= \begin{cases}1 & \text { if } j \neq 0 \text { nor } N \\ \frac{1}{\sqrt{2}} & \text { if } j=0 \text { or } N\end{cases}
$$

where $N$ is the block length of the transform. For the case when the input signal is a stream of samples, $c(n, k)$ and $s(n, k)$ are referred to as the running DCT's and DST's at time $n$. When a new sample $x(n+$ 1 ) becomes available, the signal block of interest shifts one sample in time to include $x(n+1)$ and exclude the sample $x(n-N+1)$. In this case, the running DCT's at the instant $n+1$ is determined by the following expressions [6]:

DCT-I:

$$
\begin{aligned}
c(n+1, k) & \\
= & A_{k} c(n, k)+P_{k} B_{k} s(n, k)+\sqrt{\frac{2}{N}} P_{k} \\
& \cdot\left\{-\frac{1}{\sqrt{2}} A_{k} x(n-N)+\left(\frac{1}{\sqrt{2}}-1\right) x(n-N+1)\right. \\
& \left.+(-1)^{k}\left(1-\frac{1}{\sqrt{2}}\right) A_{k} x(n)+(-1)^{k} \frac{1}{\sqrt{2}} x(n+1)\right\}
\end{aligned}
$$

DST-I:

$$
\begin{aligned}
s(n+1, k)= & A_{k} s(n, k)-B_{k} c(n, k)+\sqrt{\frac{2}{N}} B_{k} \\
& \cdot\left\{\frac{1}{\sqrt{2}} x(n-N)+\left(1-\frac{1}{\sqrt{2}}\right)(-1)^{k} x(n)\right\}
\end{aligned}
$$

\section{DCT-II:}

$$
\begin{aligned}
c(n+1, k)= & A_{k} c(n, k)+B_{k} s(n, k) \\
& +\sqrt{\frac{2}{N}} P_{k} C_{k}\left\{(-1)^{k} x(n)-x(n-N)\right\}
\end{aligned}
$$

and

DST-II:

$$
\begin{aligned}
s(n+1, k)= & A_{k} s(n, k)-B_{k} c(n, k) \\
& +\sqrt{\frac{2}{N}} P_{k} D_{k}\left\{x(n-N)-(-1)^{k} x(n)\right\}
\end{aligned}
$$

DCT-III:

$$
\begin{aligned}
c(n+1, k) & \\
= & E_{k} c(n, k)+F_{k} s(n, k)+\sqrt{\frac{2}{N}} \\
\cdot & \left\{-\frac{1}{\sqrt{2}} E_{k} x(n-N)+\left(\frac{1}{\sqrt{2}}-1\right) x(n-N+1)\right. \\
& \left.+(-1)^{k}\left(1-\frac{1}{\sqrt{2}}\right) F_{k} x(n)\right\} .
\end{aligned}
$$

DCT-III:

$$
\begin{aligned}
s(n+1, k) & \\
= & E_{k} s(n, k)-F_{k} c(n, k)+\sqrt{\frac{2}{N}} \\
\cdot & \left\{\frac{1}{\sqrt{2}} F_{k} x(n-N)+(-1)^{k}\left(1-\frac{1}{\sqrt{2}}\right) E_{k} x(n)\right. \\
& \left.+\frac{(-1)^{k}}{\sqrt{2}} x(n+1)\right\}
\end{aligned}
$$

DCT-IV:

DCT-IV:

$$
\begin{aligned}
c(n+1, k)= & E_{k} c(n, k)+F_{k} s(n, k)+\sqrt{\frac{2}{N}} \\
& \cdot\left\{-G_{k} x(n-N)+(-1)^{k} H_{k} x(n)\right\} .
\end{aligned}
$$

$$
\begin{aligned}
s(n+1, k)= & E_{k} s(n, k)-F_{k} c(n, k)+\sqrt{\frac{2}{N}} \\
& \cdot\left\{H_{k} x(n-N)+(-1)^{k} G_{k} x(n)\right\}
\end{aligned}
$$

where

$$
\begin{aligned}
& A_{k}=\cos \frac{k \pi}{N} \\
& B_{k}=\sin \frac{k \pi}{N} \\
& C_{k}=\cos \frac{k \pi}{2 N} \\
& D_{k}=\sin \frac{k \pi}{N} \\
& E_{k}=\cos \frac{(2 k+1) \pi}{2 N} \\
& F_{k}=\sin \frac{(2 k+1) \pi}{2 N} \\
& G_{k}=\cos \frac{(2 k+1) \pi}{4 N} \\
& H_{k}=\sin \frac{(2 k+1) \pi}{4 N} .
\end{aligned}
$$

Equations (9)-(12) were derived by Yip and Rao [6] and were called the shift properties, which can be used to update the transform coefficients. Consider the DCT-II as a particular example. Clearly (10a) can be used to update the DCT-II coefficients. However, the updated DCT-II is related to the DST- II coefficients. In other words, updating 
the DCT-II also requires the updating of DST-II, and this can be performed by (10b). Consequently, both DCT-II and DST-II must be updated at the same time, even in the case when only one of them is required. This problem exists for other transforms as well and is a source of excessive computational burden.

\section{SECOND-ORDER SHIFT PROPERTIES OF DCT's AND DST's}

As mentioned in Section I it is desirable to derive recursive equations which enable the independent updating of DCT's and DST's, respectively. We present the derivation for DCT-II and DST-II in detail. Similar derivations can be performed for the remaining transforms and the final results will be listed without proof in the interest of brevity.

Let us consider the DCT-II and DST-II. The objective is to derive two independent second-order one-variable recursive equations which are equivalent to (10a) and (b). We begin by taking the $z$ transform of (10a) and (b) as follows:

$$
\begin{aligned}
z C(z, k)= & A_{k} C(z, k)+B_{k} S(z, k)+\sqrt{\frac{2}{N}} P_{k} C_{k} \\
& \cdot\left[(-1)^{k}-z^{-N}\right] X(z)
\end{aligned}
$$

and

$$
\begin{aligned}
z S(z, k)= & A_{k} S(z, k)-B_{k} C(z, k)+\sqrt{\frac{2}{N}} P_{k} D_{k} \\
& \cdot\left[z^{-N}-(-1)^{k}\right] X(z)
\end{aligned}
$$

where $C(z, k), S(z, k)$, and $X(z)$ are the $z$ transforms of $c(n, k)$, $s(n, k)$ and $x(n)$, respectively. From (13a) and (13b) we can obtain

$$
\begin{aligned}
C(z, k)= & \frac{B_{k} z^{-1}}{1-A_{k} z^{-1}} S(z, k) \\
& +\frac{\sqrt{\frac{2}{N}} P_{k} C_{k}\left[(-1)^{k}-z^{-N}\right] z^{-1}}{1-A_{k} z^{-1}} X(z)
\end{aligned}
$$

and

$$
\begin{aligned}
S(z, k)= & \frac{-B_{k} z^{-1}}{1-A_{k} z^{-1}} C(z, k) \\
& +\frac{\sqrt{\frac{2}{N}} P_{k} D_{k}\left[z^{-N}-(-1)^{k}\right] z^{-1}}{1-A_{k} z^{-1}} X(z) .
\end{aligned}
$$

Substituting (14a) into (14b) and after some manipulation we have

$$
\begin{aligned}
(1 & \left.-2 A_{k} z^{-1}+z^{-2}\right) C(z, k) \\
= & \sqrt{\frac{2}{N}} P_{k} C_{k} \\
& \cdot\left[(-1)^{k} z^{-1}-(-1)^{k} z^{-2}-z^{-(N+1)}+z^{-(N+2)}\right] X(z) .
\end{aligned}
$$

In the same way, substituting (14b) into (14a) and after some manipulation we have

$$
\begin{aligned}
(1- & \left.2 A_{k} z^{-1}+z^{-2}\right) S(z, k) \\
= & \sqrt{\frac{2}{N}} P_{k} D_{k} \\
& \cdot\left[(-1)^{k} z^{-1}-(-1)^{k} z^{-2}+z^{-(N+1)}+z^{-(N+2)}\right] X(z) .
\end{aligned}
$$

Taking the inverse $z$ transform of (15a) and (15b) yields

$$
\begin{aligned}
c(n+1, k)=2 & A_{k} c(n, k)-c(n-1, k)+T_{2,1}^{k} \\
\cdot & {\left[(-1)^{k} x(n)-(-1)^{k} x(n-1)\right.} \\
& -x(n-N)+x(n-N-1)]
\end{aligned}
$$

and

$$
\begin{array}{r}
s(n+1, k)=2 A_{k} s(n, k)-s(n-1, k)+U_{2,1}^{k} \\
\cdot\left[-(-1)^{k} x(n)-(-1)^{k} x(n-1)\right. \\
\quad+x(n-N)+x(n-N-1)]
\end{array}
$$

where

$$
T_{2,1}^{k}=\sqrt{\frac{2}{N}} P_{k} C_{k} \quad \text { and } \quad U_{2,1}^{k}=\sqrt{\frac{2}{N}} P_{k} D_{k} .
$$

Equations (16) and (17) represent the second-order shift properties for DCT-II and DST-II. Close examination of (16) and (17) indicate that they are independent of each other and, hence, can be used to recursively update the DCT-II and DST-II, respectively. It is interesting to note that there are similarities between (16) and (17) and the frequency sampling structure for FIR filter implementation [9].

The second-order shift properties for other members of the DCT and DST families can be obtained using a similar approach and the results are listed as follows:

DCT-I:

$$
\begin{aligned}
c(n+ & 1, k) \\
= & 2 A_{k} c(n, k)-\left[A_{k}^{2}+P_{k} B_{k}^{2}\right] c(n-1, k) \\
& +T_{1,1}^{k} x(n+1)+T_{1,2}^{k} x(n)+T_{1,3}^{k} x(n-1) \\
& -T_{1,4}^{k} x(n-N+1)+T_{1,5}^{k} x(n-N) \\
& +T_{1,6}^{k} x(n-N-1)
\end{aligned}
$$

where

$$
\begin{aligned}
& T_{1,1}^{k}=(-1)^{k} \sqrt{\frac{1}{2}} \\
& T_{1,2}^{k}=(-1)^{-k}(1-\sqrt{2}) A_{k} P_{k} \sqrt{\frac{2}{N}} \\
& T_{1,3}^{k}=-(-1)^{-k}\left(1-\sqrt{\frac{1}{2}}\right) P_{k} \sqrt{\frac{2}{N}} \\
& T_{1,4}^{k}=\left(\sqrt{\frac{1}{2}}-1\right) P_{k} \sqrt{\frac{2}{N}} \\
& T_{1,5}^{k}=(1-\sqrt{2}) A_{k} P_{k} \sqrt{\frac{2}{N}}
\end{aligned}
$$

and

$$
T_{1,6}^{k}=P_{k} \sqrt{\frac{1}{N}}
$$

Note that in (18) the coefficient, $\left[A_{k}^{2}+P_{k} B_{k}^{2}\right]$, is always equal to one when $k \neq 0$ and $k \neq N$. This means that this coefficient introduces a multiplication only when $k=0$ or $k=N$

DST-I:

$$
\begin{aligned}
& s(n+1, k) \\
& \quad=2 A_{k} s(n, k)-\left[A_{k}^{2}+P_{k} B_{k}^{2}\right] s(n-1, k) \\
& \quad+U_{1,1}^{k} x(n)+U_{1,2}^{k} x(n-1)+U_{1,3}^{k} x(n-N) \\
& \quad+U_{1,4}^{k} x(n-N-1)
\end{aligned}
$$

where

$$
\begin{aligned}
& U_{1,1}^{k}=(-1)^{-k} B_{k}\left(\frac{1-P_{k}}{\sqrt{2}}-1\right) \sqrt{\frac{2}{N}} \\
& U_{1,2}^{k}=(-1)^{k}\left(1-\sqrt{\frac{1}{2}}\right) A_{k}\left(1-P_{k}\right) B_{k} \sqrt{\frac{2}{N}} ; \\
& U_{1,3}^{k}=B_{k}\left(\sqrt{\frac{1}{2}}+\left(1-\sqrt{\frac{1}{2}}\right) P_{k}\right) \sqrt{\frac{2}{N}}
\end{aligned}
$$

and

$$
U_{1,4}^{k}=A_{k} B_{k}\left(P_{k}-1\right) \sqrt{\frac{1}{N}} .
$$


DCT-III:

$$
\begin{aligned}
& c(n+1, k) \\
& =2 E_{k} c(n, k)-c(n-2, k)+T_{3,1}^{k} x(n) \\
& \quad+T_{3,2}^{k} x(n-N+1)+T_{3,3}^{k} x(n-N) \\
& \quad+T_{3,4}^{k} x(n-N-1)
\end{aligned}
$$

where

$$
\begin{aligned}
T_{3,1}^{k} & =(-1)^{k} \sqrt{\frac{2}{N}} F_{k} \\
T_{3,2}^{k} & =\left(1-\sqrt{\frac{1}{2}}\right) \sqrt{\frac{2}{N}} \\
T_{3,3}^{k} & =(1-\sqrt{2}) E_{k} \sqrt{\frac{2}{N}}
\end{aligned}
$$

and

$$
T_{3,4}^{k}=\sqrt{\frac{1}{N}}
$$

\section{DST-III:}

$$
\begin{aligned}
s(n+1, k)= & 2 E_{k} s(n, k)-s(n-1, k)+U_{3,1}^{k} x(n+1) \\
& +U_{3,2}^{k} x(n)+U_{3,3}^{k} x(n-1)+U_{3,4}^{k} x(n-N)
\end{aligned}
$$

where

$$
\begin{aligned}
U_{3,1}^{k} & =(-1)^{k} \sqrt{\frac{1}{N}} \\
U_{3,2}^{k} & =(-1)^{k} \sqrt{\frac{2}{N}}\left[\left(1-\sqrt{\frac{1}{2}}\right) F_{k}-\sqrt{\frac{1}{2}} E_{k}\right] \\
U_{3,3}^{k} & =-(-1)^{k}\left(1-\sqrt{\frac{1}{2}}\right) \sqrt{\frac{2}{N}}\left(F_{k}^{2}+E_{k} F_{k}\right)
\end{aligned}
$$

and

$$
U_{3,4}^{k}=-\sqrt{\frac{1}{N}} F_{k}
$$

DCT-IV:

$$
\begin{aligned}
c(n+ & 1, k) \\
= & 2 E_{k} c(n, k)-c(n-1, k) \\
& +T_{4,1}^{k}[x(n)+x(n-1)] \\
& +T_{4,2}^{k}[x(n-N)-x(n-N-1)]
\end{aligned}
$$

where

and

$$
T_{4,1}^{k}=(-1)^{k} \sqrt{\frac{2}{N}} H_{k}
$$

$$
T_{4,2}^{k}=-G_{k} \sqrt{\frac{2}{N}}
$$

DST-IV:

$$
\begin{aligned}
& s(n+1, k) \\
& \quad=2 E_{k} s(n, k)-s(n-1, k)+U_{4,1}^{k}[x(n)-x(n-1)] \\
& \quad+U_{4,2}^{k}[x(n-N)-x(n-N-1)]
\end{aligned}
$$

where

$$
U_{4,1}^{k}=(-1)^{k} G_{k} \sqrt{\frac{2}{N}} \text { and } U_{4,2}^{k}=H_{k} \sqrt{\frac{2}{N}} .
$$

Equations (16)-(29) provide a new approach for implementing a running DCT and DST algorithm.
TABLE I

COMPARISON OF COMPUTATIONAL BURDEN

\begin{tabular}{l|l|c|l|l|l|l}
\hline \multirow{2}{*}{ Transforms } & \multicolumn{3}{|c|}{ Multiplicat ions } & \multicolumn{3}{c}{ Additions } \\
\cline { 2 - 8 } & Ast Order & Ref [7] & $\begin{array}{l}\text { Proposed } \\
\text { Approach }\end{array}$ & $\begin{array}{l}\text { 1st Order } \\
\text { Approach }\end{array}$ & Ref [7] & $\begin{array}{l}\text { Proposed } \\
\text { Approach }\end{array}$ \\
\hline DCT-I & $10 \mathrm{~N}$ & & $7 \mathrm{~N}$ & $8 \mathrm{~N}$ & & $7 \mathrm{~N}$ \\
\hline DCT-II & $6 \mathrm{~N}$ & $5 \mathrm{~N}$ & $2 \mathrm{~N}$ & $4 \mathrm{~N}+2$ & $5 \mathrm{~N}+2$ & $2 \mathrm{~N}+4$ \\
\hline DCT-III & $10 \mathrm{~N}$ & & $5 \mathrm{~N}$ & $8 \mathrm{~N}$ & & $5 \mathrm{~N}$ \\
\hline DCT-IV & $8 \mathrm{~N}$ & $6 \mathrm{~N}$ & $3 \mathrm{~N}$ & $6 \mathrm{~N}$ & $8 \mathrm{~N}$ & $2 \mathrm{~N}+2$ \\
\hline DST-I & $10 \mathrm{~N}$ & & $5 \mathrm{~N}$ & $8 \mathrm{~N}$ & & $5 \mathrm{~N}$ \\
\hline DST-II & $6 \mathrm{~N}$ & $5 \mathrm{~N}$ & $2 \mathrm{~N}$ & $4 \mathrm{~N}+2$ & $5 \mathrm{~N}+2$ & $2 \mathrm{~N}+4$ \\
\hline DST-III & $10 \mathrm{~N}$ & & $5 \mathrm{~N}$ & $8 \mathrm{~N}$ & & $5 \mathrm{~N}$ \\
\hline DST-IV & $8 \mathrm{~N}$ & $6 \mathrm{~N}$ & $3 \mathrm{~N}$ & $6 \mathrm{~N}$ & $8 \mathrm{~N}$ & $2 \mathrm{~N}+2$ \\
\hline
\end{tabular}

TABLE II

COMPARISON OF MEMORY LOCATIONS

\begin{tabular}{l|c|c|c|c|c|c|c|c}
\hline Transforms & \multicolumn{2}{|c|}{ Coefficients } & \multicolumn{2}{c|}{$\begin{array}{l}\text { Transform } \\
\text { domain data }\end{array}$} & \multicolumn{2}{c|}{ Input sequence } & \multicolumn{2}{c}{ Overall comparison } \\
\cline { 2 - 9 } & $\begin{array}{l}1^{\text {st }} \text { order } \\
\text { approach }\end{array}$ & $\begin{array}{l}2^{\text {nd }} \text { order } \\
\text { approach }\end{array}$ & $\begin{array}{c}1^{\text {st }} \text { order } \\
\text { approach }\end{array}$ & $\begin{array}{l}2^{\text {nd }} \text { order } \\
\text { approach }\end{array}$ & $\begin{array}{l}1^{\text {st }} \text { order } \\
\text { approach }\end{array}$ & $\begin{array}{l}2^{\text {nd }} \text { approach } \\
\text { approach }\end{array}$ & $\begin{array}{l}1^{\text {st }} \text { order } \\
\text { approach }\end{array}$ & $\begin{array}{l}2^{\text {nd }} \text { order } \\
\text { approach }\end{array}$ \\
\hline DCT-I & $8 \mathrm{~N}$ & $8 \mathrm{~N}$ & $2 \mathrm{~N}$ & $2 \mathrm{~N}$ & $\mathrm{~N}+2$ & $\mathrm{~N}+3$ & $11 \mathrm{~N}+2$ & $11 \mathrm{~N}+3$ \\
\hline DCT-II & $4 \mathrm{~N}$ & $2 \mathrm{~N}$ & $2 \mathrm{~N}$ & $2 \mathrm{~N}$ & $\mathrm{~N}+1$ & $\mathrm{~N}+2$ & $7 \mathrm{~N}+3^{*}$ & $5 \mathrm{~N}+4^{*}$ \\
\hline DCT-III & $8 \mathrm{~N}$ & $5 \mathrm{~N}$ & $2 \mathrm{~N}$ & $2 \mathrm{~N}$ & $\mathrm{~N}+2$ & $\mathrm{~N}+2$ & $11 \mathrm{~N}+2$ & $8 \mathrm{~N}+2$ \\
\hline DCT-IV & $4 \mathrm{~N}$ & $3 \mathrm{~N}$ & $2 \mathrm{~N}$ & $2 \mathrm{~N}$ & $\mathrm{~N}+1$ & $\mathrm{~N}+2$ & $7 \mathrm{~N}+1$ & $6 \mathrm{~N}+4^{*}$ \\
\hline DST-I & $8 \mathrm{~N}$ & $6 \mathrm{~N}$ & $2 \mathrm{~N}$ & $2 \mathrm{~N}$ & $\mathrm{~N}+2$ & $\mathrm{~N}+2$ & $11 \mathrm{~N}+2$ & $9 \mathrm{~N}+2$ \\
\hline DST-II & $4 \mathrm{~N}$ & $2 \mathrm{~N}$ & $2 \mathrm{~N}$ & $2 \mathrm{~N}$ & $\mathrm{~N}+1$ & $\mathrm{~N}+2$ & $7 \mathrm{~N}+3^{*}$ & $5 \mathrm{~N}+4^{*}$ \\
\hline DST-III & $8 \mathrm{~N}$ & $5 \mathrm{~N}$ & $2 \mathrm{~N}$ & $2 \mathrm{~N}$ & $\mathrm{~N}+2$ & $\mathrm{~N}+2$ & $11 \mathrm{~N}+2$ & $8 \mathrm{~N}+2$ \\
\hline DST-IV & $4 \mathrm{~N}$ & $3 \mathrm{~N}$ & $2 \mathrm{~N}$ & $2 \mathrm{~N}$ & $\mathrm{~N}+1$ & $\mathrm{~N}+2$ & $7 \mathrm{~N}+2$ & $6 \mathrm{~N}+4^{*}$ \\
\hline
\end{tabular}

\section{PERformance AnAlysis}

The computation of running DCT's and DST's based on SOS properties is expected to provide certain advantages over the first-order approach proposed by Yip and Rao [6] as well as the algorithm proposed by Murthy and Swamy [7].

Let us first compare the computational burden associated with the proposed approaches against those by Yip and Rao [6] and Murthy and Swamy [7]. Taking DCT-II as the key example, from (9a) and (b) it is clear that the first-order approach needs the following computation:

- $6 N$ multiplications with coefficients;

- $4 N$ addition of terms;

- 2 additions for $2\left[(-1)^{k} x(n)-x(n-N)\right]$ for even and odd $k$. Similarly, from (16) the proposed second-order approach requires the following operations:

- $2 N$ multiplications with coefficients;

- $2 N$ additions of terms;

- four additions for

$$
\left[(-1)^{k}[x(n)-x(n-1)]-x(n-N)+x(n-N-1)\right]
$$

for odd and even $k$.

Hence, for DCT-II the second-order approach can reduce the number of multiplications by $67 \%$ when compared with the first-order approach. Table I lists the results for all the transform members. Clearly, in all cases the computational burden associated with the proposed approaches is significantly reduced when compared to both references [6] and [7].

Now let us consider the memory requirements. Note that in the case of [7] there is no improvement in terms of the memory requirements when compared with the first-order approach [6]. This is because both the real and imaginary part of the complex function need storage locations. Hence, we only compare the proposed algorithm with the firstorder approach [6]. For both algorithms, memory is required to store 1) the coefficients of the recursive equations; 2) transform results; and 3) the input signal samples. Table II depicts the comparison between these two methods. As expected, the memory requirements for the proposed approach are either equal to or better than the first-order algorithm [6]. 


\section{CONCLUSION}

In this paper, we have derived the SOS properties for DCT's and DST's. The result is a new approach for computing the running DCT's and DST's. The computational burden and memory requirements associated with the proposed approach have been investigated and compared with the first-order approach proposed by Yip and Rao [6], as well as the approach proposed by Murthy and Swamy [7]. It has been shown that the proposed approach is characterized by reduced computational burden and generally lower memory storage requirements.

\section{REFERENCES}

[1] N. Ahmed, T. Natarajan, and K. R. Rao, "Discrete cosine transform," IEEE Trans. Comput., vol. C-23, pp. 90-94, Jan. 1974.

[2] W. H. Chen, C. H. Smith, and S. C. Fralick, "A fast computational algorithm for the discrete cosine transform," IEEE Trans. Commun., vol. COM-25, pp. 1004-1009, Sept. 1977.

[3] B. G. Lee, "A new algorithm to compute the discrete cosine transform," IEEE Trans. Acoust., Speech, Signal Processing, vol. ASSP-32, pp. 1243-1245, Dec. 1984.
[4] M. Malvar, "Fast computation of discrete cosine transform through fast Hartley transform," Electron. Lett., vol. 22, no. 7, pp. 352-353, March 1986.

[5] Y.-H. Chan and W.-C. Siu, "Mixed-radix discrete cosine transform," IEEE Trans. Signal Processing, vol. 41, pp. 3157-3161, Nov. 1993.

[6] P. Yip and K. R. Rao, "On the shift properties of DCT's and DST's," IEEE Trans. Acoust., Speech, Signal Processing, vol. 35, pp. 404-406, Mar. 1987.

[7] N. R. Murthy and M. N. S. Swamy, "On the computation of running discrete Cosine and Sine transforms," IEEE Trans. Signal Processing, vol. 40, pp. 1430-1437, June 1992.

[8] Z. Wang, "Fast algorithms for the discrete $W$ transform and for the discrete Fourier transform," IEEE Trans. Acoust., Speech, Signal Processing, vol. ASSP-32, pp. 803-816, Aug. 1984.

[9] A. V. Oppenheim and R. W. Schafer, Digital Signal Processing. Englewood Cliffs, NJ: Prentice-Hall, 1975. 